\title{
Face Recognition Using Simplicial Complexes
}

\author{
Chengming Zou ${ }^{1}$ and Edwin R. Hancock ${ }^{2}$ \\ 1 School of Computer Science, Wuhan University of Technology, \\ Wuhan, Hubei, 430070, China \\ zoucm@hotmail.com \\ 2 Department of Computer Science, The University of York, York, YO10 5DD, UK \\ erh@cs.york.ac.uk
}

\begin{abstract}
The paper presents a novel method for 3D facial shape recognition. Our inputs are 3D facial shapes which are reconstructed from point clouds, and then filtered using PCA. The resulting data are represented by simplicial complexes. This representation can capture topological and geometric information at a specified resolution with a small number of control points. We calculate the Gromov-Hausdorff distance between simplicial complexes, and this measures how far each pair of faces are from being isometric. Finally, we demonstrate our method in an application to point clouds collected from laser range scanner.
\end{abstract}

Keywords: Simplicial Complexes, Gromov-Hausdorff Distance, 3D Facial Shape, Recognition.

\section{Introduction}

Face recognition is one of the most significant applications of image understanding. Although rapid progress has been made in this area during the last few years, the general task of recognition remains unsolved. In general, face appearance is influenced not only by intrinsic factors such as shape and texture (albedo), but also by extrinsic ones such as illumination and viewpoint. As a result there have been attempts to compute facial shape invariants that can be used to recognise a face. It has been shown that statistical models based on the distribution of surface normals can offer a powerful means of representing and recognising facial shape. For instance, Smith and Hancock [1] project the surface normals into a tangent space to construct a statistical model using principal geodesic analysis. This work has recently been extended to gender recognition [2], but has proved too cumbersome for expression recognition. Bronstein, Bronstein and Kimmel [3], on the other hand, develop a spherical embedding, that allows faces to be represented in a manner that is invariant to expression. Parameterizing the distribution of surface normals, Kazhdan et al. 4] use the fact that the spherical harmonics of a given frequency form a subspace which is a rotationally invariant. This method can be applied to the extended Gaussian image (EGI) to create a rotationally invariant shape descriptor.

Recently, it has been shown that topological methods provide a robust approach for shape comparison and 3D object recognition, which can also be used

J.A. Carrasco-Ochoa et al. (Eds.): MCPR 2010, LNCS 6256, pp. 22-29, 2010.

(C) Springer-Verlag Berlin Heidelberg 2010 
for face recognition. Singh, Mmoli and Carlsson [5] use the "Mapper" system for extracting simple descriptions of high dimensional data sets in the form of simplicial complexes. They implement 3D object recognition by comparing the distance between two simplicial complexes. Mmoli and Sapiro [6] present a theoretical and computational framework for isometry invariant recognition of point cloud data based on Gromov-Hausdorff distances. Our aim in this paper is to explore whether such topological descriptions can be used for view and expression invariant face recognition. We commence by converting facial range-data into a simplicial complex and then use the Gromov-Hausdorff distance as a similarity measure.

The outline of the paper is as follows. We commence in section 2 by describing preprocessing the raw data. Section 3 details how to represent the facial shape via simplicial complexes. In section 4 we describe how to recognise face by calculate the Gromov-Hausdorff distance. Experiments are presented in section 5 . Finally,section 6 concludes the paper and offer directions for future investigation.

\section{Preprocessing}

Our raw data is in the form of point clouds collected using a Cyberware 3030 laser range scanner, which is capable of digitising the surface of objects with a resolution of up to $1024 \times 1440$ points. We use PCA to remove noise from the point cloud data. Let $p_{i}\left(x_{i}, y_{i}, z_{i}\right)$ denote the $i^{\text {th }}$ point of a point cloud which has $N$ points. Let $\bar{p}=\frac{1}{N} \sum_{i=1}^{N} p_{i}$ denote the mean coordinate. The data matrix $A=\left[\left(p_{1}-\bar{p}\right)\left(p_{2}-\bar{p}\right) \cdots\left(p_{N}-\bar{p}\right)\right]$, is constructed by subtracting the mean coordinate. Then the covariance matrix of the data is $C=A A^{T}$, which is a $3 \times 3$ matrix. Performing an eigen-decomposition on $C$, yields the eigenvectors $v_{1}, v_{2}, v_{3}$ and their corresponding eigenvalues $\lambda_{1}, \lambda_{2} \lambda_{3}$, where $\lambda_{1}>\lambda_{2}>\lambda_{3}$. The first eigenvector gives us the leading principal component of the point cloud, and the last eigenvector the least important variance component, which we consider as noise in the point cloud. We therefore use $v_{3}$ to remove the noise component NoiseComponent $=v_{3} \times A^{T}$, denoted as $P^{\prime}=\left\{p_{1}^{\prime}, p_{2}^{\prime}, \cdots, p_{N}^{\prime}\right\}, p_{i}^{\prime}=$ $\left(x_{i}^{\prime}, y_{i}^{\prime}, z_{i}^{\prime}\right), i=1,2, \cdots, N$ from the raw data. Then we can find the minimum or maximum value (extrema) of $z_{i}^{\prime}, i=1,2, \cdots, N$ and its corresponding location, denoted as $z_{k}^{\prime}$ and $p_{k}^{\prime}$ respectively. From the extremum location can construct the neighbourhood $N B$ of $p_{k}^{\prime}$, which contains points that are both close to $p_{k}^{\prime}$ and have $z$ coordinates with the same sign as $z_{k}^{\prime}$. Removing those points in the neighbourhood $N B$ will implement remove the noisy points from the point cloud.

\section{Facial Shape Representation Using Simplicial Complexes}

An obvious way to convert a point cloud residing in a metric space into a global object is to use the set of points in the cloud as the vertices of a combinatorial 
graph whose edges are determined by proximity (i.e. whether vertices are within some specified distance of one-another) [7. Such a graph, while capturing connectivity information, ignores a wealth of higher order relational features which go beyond defining clusters. We can interpret these features by thinking of the graph as a scaffold for a higher-dimensional object. So to go from a point cloud to a simplicial complex is to embed the point cloud in a piecewise space constructed from simplicies identified (combinatorially) with the faces of the graph. The freedom of choice as to how to fill in the higher dimensional simplicies of the proximity graph allows for different global representations. A natural method for so doing is using $\breve{C}$ ech theory [7]. To do so we make use of the Rips complex, which is the abstract simplicial complex whose $k$-simplices correspond to unordered $(k+1)$-tuples of the point cloud which are within a pairwise distance $\epsilon$. Although the Rips complex has more simplices than alternative representations, it is less expensive from a computational point of view. The reason is that the Rips complex is a flag complex, i.e. it is maximal among all simplicial complexes with the given 1-skeleton. Thus, the combinatorics of the 1-skeleton completely determines the complex.

Motivated by the $\breve{C}$ ech theory, we commence our construction of the simplicial complex representation by firstly locating a covering for the given metric space. Given a point cloud, it is not straightforward to directly locate such a covering. However, for a closed interval $Z=[a, b] \subseteq R$, we may easily locate a covering. Now suppose that we are given a metric space possessing a continuous map $f: X \rightarrow Z$ onto $Z$, and that $Z$ possesses a covering $\mathcal{U}=\left\{U_{\alpha}\right\}_{\alpha \in A}$, again for some finite indexing set $A$. Since $f$ is continuous, the set $f^{-1}\left(U_{\alpha}\right)$ also forms an open covering of $X$. For each $\alpha$, we can now consider the decomposition of $f^{-1}\left(U_{\alpha}\right)$ into its path connected components. So we write $f^{-1}\left(U_{\alpha}\right)=\bigcup_{i=1}^{j_{\alpha}} V(\alpha, i)$, where $j_{\alpha}$ is the number of connected components in $f^{-1}\left(U_{\alpha}\right)$. That is to say, we have located a covering $f^{-1}\left(U_{\alpha}\right)$ for $X$, and we refer to $f$ as a filter.

Our aim in this paper is to represent facial shape using simplicial complexes. In other words, we reduce high dimensional data sets into simplicial complexes with a significant reduction in the number of points needed to represent the topological and geometric information at a specified resolution. To this end, we subsample from the facial shape to reduce the number of points. We then cluster the remaining points using the available geodesic distance matrix and the self organising map (SOM). Then we can construct a simplicial complex by adding a $n-1$-simplex to it whenever $n$ clusters have non-empty intersection.

\section{Dissimilarity Calculation}

In this section we show how simplicial complexes can be used for facial shape representation and recognition. From the above discussion, the process of face recognition is equivalent to searching for isometric simplicial complexes using the Gromov-Hausdorff. From [8], every facial shape has a "Point fingerprint", which 
is the set of iso-depth contours around the nose tip. Since faces are of different size, we do not need to measure the whole facial shape. Instead we need only measure the point fingerprint region. If two point fingerprints are isometric, then the corresponding facial shapes correspond to the same individual with the same or different expression. So to recognise a face, we need only to calculate a sub graph corresponding to the point fingerprint region. We refer to this sub graph as the fingerprint graph.

Consider two fingerprint graphs $G_{1}\left(V_{1}, E_{1}\right)$ and $G_{2}\left(V_{2}, E_{2}\right)$. From the above preprocessing steps, the two fingerprint graphs form a sub-space of the spherical metric space. As a result the Gromov-Hausdorff distance between $G_{1}$ and $G_{2}$ is

$$
d_{\mathcal{G H}}\left(G_{1}, G_{2}\right)=\inf \max \left\{\sup _{u \in V_{1}} \inf _{v \in V_{2}} d(u, v), \sup _{v \in V_{2}} \inf _{u \in V_{1}} d(u, v)\right\}
$$

The Gromov-Hausdorff distance $d_{\mathcal{G H}}\left(G_{1}, G_{2}\right)$ measures the dissimilarity of two facial shapes. By setting a distance threshold $\tau$, when $d_{\mathcal{G H}}\left(G_{1}, G_{2}\right) \leq \tau$ then two facial shapes are deemed to be isometric or the same facial shape with the same or different expression.
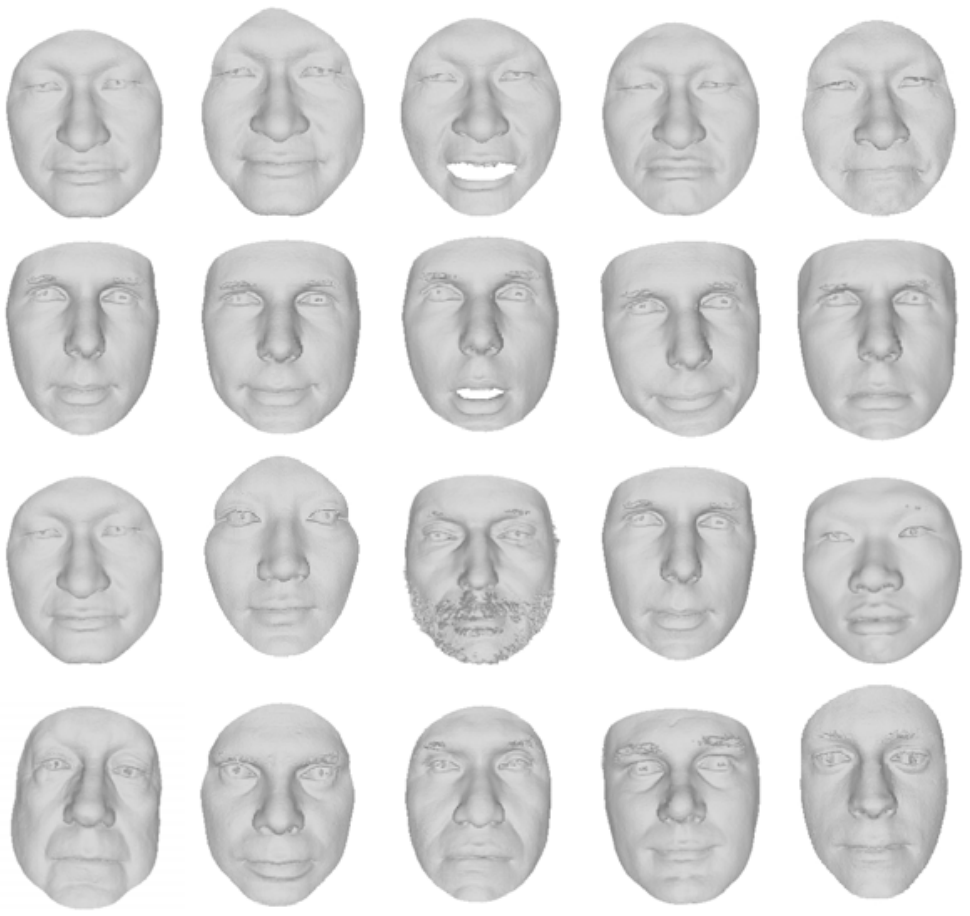

Fig. 1. Facial shape after preprocessing, first row shows facial shape A with its five expressions, the second row shows facial shape $\mathrm{D}$ with its five expressions, the third row and last row are facial shapes A,B,C,D,E,F,G,H,I,J with their neutral expression. 


\section{Experiments}

We have performed preliminary experiments on several 3D range data-sets of faces. The experiments are performed by using VS2005 and MatLab. We have performed three sets of experiments, aimed at evaluating the performance of the three steps of the method. Each experiment is performed on ten individuals, labeled with the letters A-J, who present five facial expressions (neutral, smile, laugh, sad, surprised). In each case we commence with point clouds containing 46149 vertices. After subsampling, 5829 vertices remain. A sample of the experimental results is shown in Figure 1, It is clear that the reconstruction is accurate and that our method can effectively filter noise from the data.

Experimental results with simplicial complex representations are shown in Figure 2. From Figure 2 it is clear that the facial shapes of the different subjects give rise to distinct simplicial complex representations. Moreover, the different expressions from the same individual give rise to similar simplicial complexes.

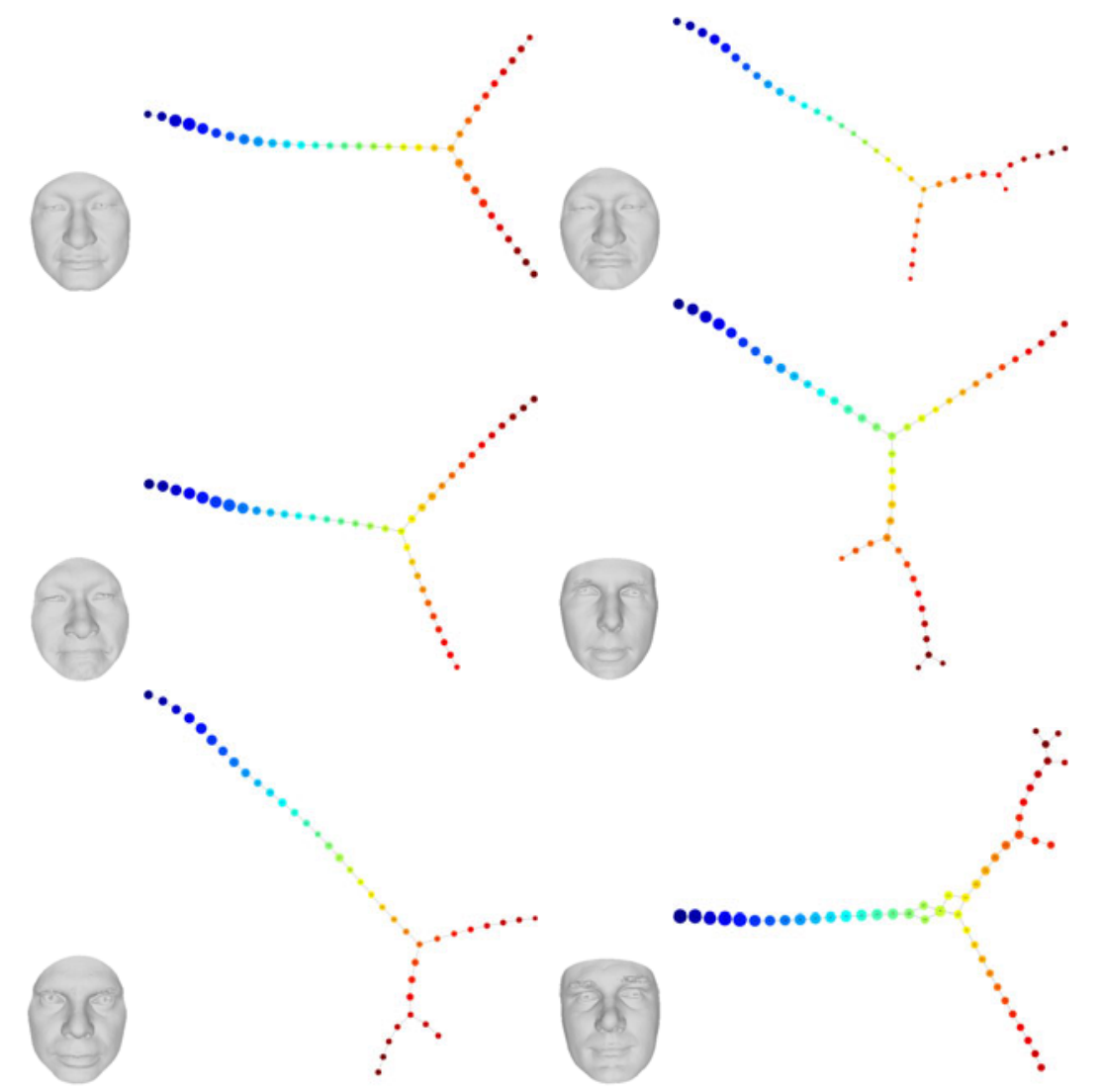

Fig. 2. Some facial shapes and their simplicial complex representation visualized by GraphViz 


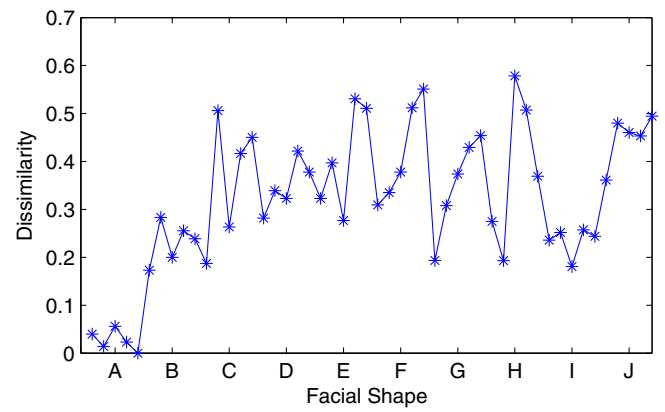

(a) Dissimilarity of facial shape A with sad expression

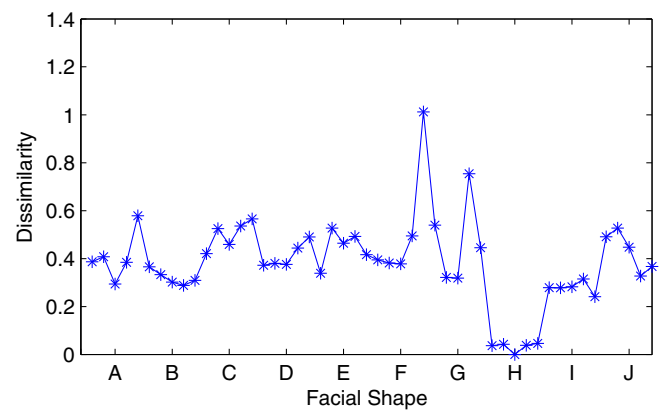

(b) Dissimilarity of facial shape $\mathrm{H}$ with laugh expression

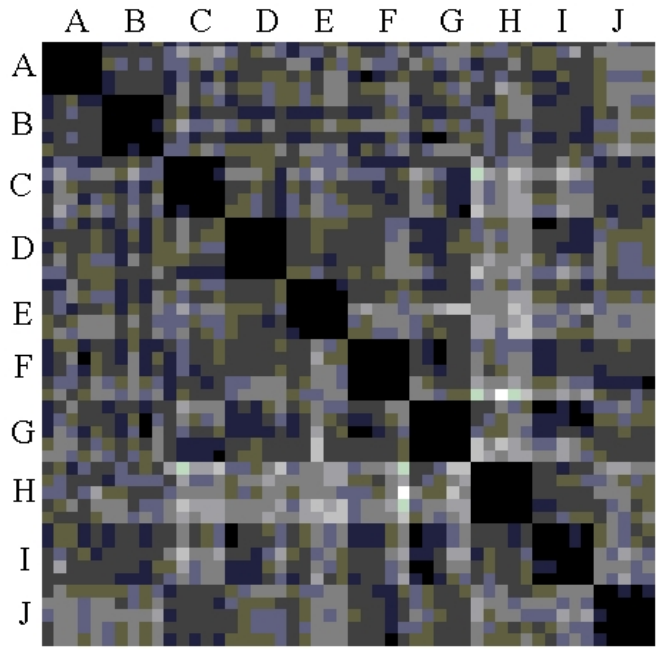

(c) The dissimilarity matrix

Fig. 3. Facial shape dissimilarity, all the dissimilarities have been divided by the maximum value 100 


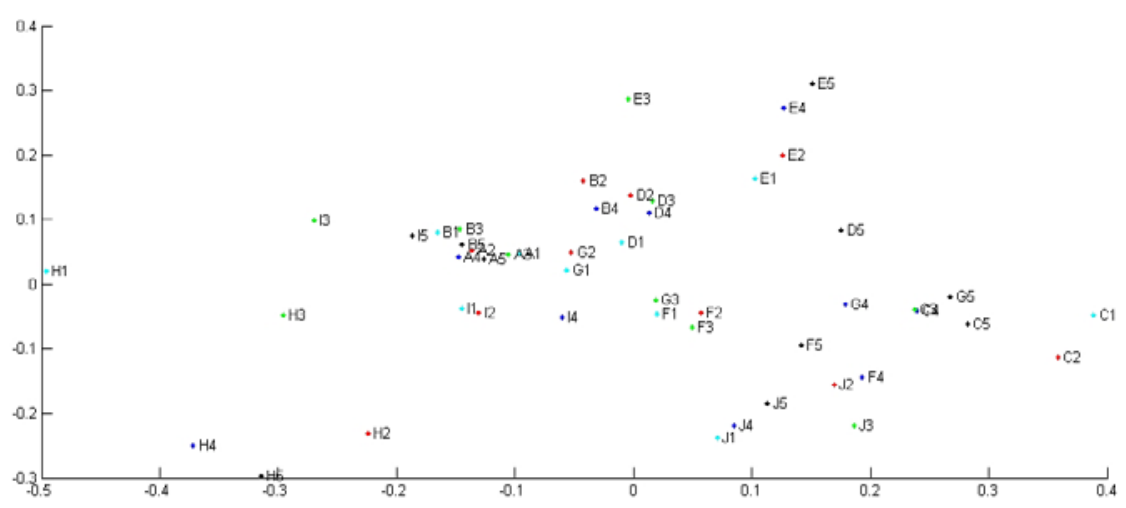

Fig. 4. The dissimilarity multidimensional scaling

That is say the simplicial complex is an effective means of expression invariant face recognition. Moreover the representation is based on just 5829 points, and this represents a data reduction factor of $15 \%$.

In Figure 3 we explore the properties of the dissimilarity measure. Figs 3a and $3 \mathrm{~b}$ show the distribution of distances for two expressions (face A with a sad expression and face $\mathrm{H}$ with a laugh expression). In both the cases the distances for the same subject are the smallest. Figure $3 \mathrm{c}$ shows the distance matrix for the complete set of similarity data. Here the different expressions for the same subject appear as distinct blocks.

To take this study one step further, in Figure 4 we embed the different expressions of the different faces into a 2 dimensional space using multidimensional scaling. Each face is denoted by a alphanumeric symbol (e.g. G2) to denote the subject and the expression. From the figure it is clear that the different expressions of the same subject project into the same region. In other words the Gromov-Hausdorff distance can be used to distinguish subjects in an expression invariant manner.

\section{Conclusions}

We have introduced a theoretical and computational framework for expression invariant facial shape representation and recognition. Experimental results have shown that our method for 3D facial shape representation and recognition is feasible and effective. Much work remains to be done in the future, especially in recognising the facial shape recovered from the photos, and verifying the validity of our method by doing more detailed experiments.

\section{References}

1. Smith, W.A.P., Hancock, E.R.: Facial Shape-from-shading and Recognition Using Principal Geodesic Analysis and Robust Statistics. Int. Journal Computer Vision 76, 71-91 (2008) 
2. Wu, J., Smith, W.A.P., Hancock, E.R.: Weighted Principal Geodesic Analysis for Facial Gender Classification. In: Rueda, L., Mery, D., Kittler, J. (eds.) CIARP 2007. LNCS, vol. 4756, pp. 331-339. Springer, Heidelberg (2007)

3. Bronstein, A., Bronstein, M., Kimmel, R.: Expression invariant face recognition via spherical embedding. Image Processing, ICIP 3, 756-759 (2005)

4. Kazhdan, M., Funkhouser, T., Rusinkiewicz, S.: Rotation Invariant Spherical harmonic representation of 3D shape descriptors. In: Proceedings of the 2003 Eurographics, vol. 43, pp. 126-164 (2003)

5. Singh, G., Mmoli, F., Carlsson, G.: Topological Methods for the Analysis of High Dimensional Data Sets and 3D Object Recognition. In: Eurographics Symposium on Point-Based Graphics (2007)

6. Mmoli, F., Sapiro, G.: A Theoretical and Computational Framework for Isometry Invariant Recognition of Point Cloud Data. Foundations of Computational Mathematics 5, 313-347 (2005)

7. Ghrist, R.: Barcodes: the persistent topology of data. In: AMS Current Events Bulletin, 2007 Joint Mathematics Meetings, New orleans, LA, January 7-8 (2007)

8. Sun, Y., Abidi, M.: Surface matching by 3d point's fingerprint. In: International Conference on Computer Vision, ICCV 2001, pp. 263-269 (July 2001) 